\title{
Review: zuclopenthixol is not more effective than other neuroleptic drugs in the acute treatment of schizophrenia
}

\author{
Fenton M, Coutinho E, Campbell C. Zuclopenthixol acetate in the treatment of acute schizophrenia and similar serious mental \\ illnesses. In: Cochrane Database of Systematic Reviews. The Cochrane Library. Oxford: Update Software; 1997: issue 4.
}

\section{Objective}

To compare the effectiveness, by systematically reviewing the literature, of zuclopenthixol acetate with other neuroleptic drugs in the acute treatment of schizophrenia and similar serious mental illnesses.

\section{Data sources}

Studies were identified by searching the Cochrane Schizophrenia Group's Register of Trials, The Cochrane Library, Medline, and abstracts of congresses and trial reference lists. Authors of relevant studies were contacted, and an appeal for unpublished data was sent out to the research community and the medical information department of Lundbeck Limited.

\section{Study selection}

Studies were selected if they were randomised controlled trials comparing zuclopenthixol acetate with other neuroleptic drugs in patients with schizophrenia or similar psychotic illnesses. Studies that evaluated the drugs in patients with dementia, substance use disorders, or depressive disorders were excluded.

\section{Data extraction}

Data were extracted independently by 2 reviewers on mental state symptoms, sedation, side effects, behaviour, and clinical global impression. Authors were contacted for missing or additional data.

\section{Main results}

5 studies met the selection criteria. When combining data from 2 trials (only 2 trials had data that could be extracted), no difference existed for important improvement in mental state at $>36$ hours $\{\mathrm{p}=0.65\}^{*}$ (table). Different instruments were used among the studies to evaluate sedation. One study found that more patients receiving zuclopenthixol acetate (6 out of 20 ) were sedated at 4 hours than were those receiving haloperidol (1 out of 20) $\{\mathrm{p}=0.038\}^{*}$ (table). Only 1 study reported scores on the Nurses Observational Scale for Inpatient Evaluation and on clinical global impression; no statistically significant differences were found between treatments. Data on the use of additional antipsychotic medication were inconclusive and data on total number of hospital admissions were not obtainable. Side effects were poorly reported in the 5 studies but there was no evidence of a consistent difference between groups for pattern of side effects or for leaving the study early.

\section{Conclusion}

This review does not provide any evidence to show that zuclopenthixol acetate is more effective than other neuroleptic drugs in controlling aggressive or disorganised behaviour or acute psychotic symptoms or in preventing side effects.

*p value calculated from data in article.

Source of funding: West Midlands National Health Service Research and Development.

For article reprint: $\mathrm{Mr} M$ Fenton, Research Nurse, South Warwickshire Mental Health Services, St Michael's Hospital, St Michael's Road, Warwick CV34 50WUK. Fax +44 (0)1865316023.

Zuclopenthixol v standard drug care†

\begin{tabular}{|c|c|c|c|c|c|}
\hline Outcome & Zuclopenthixol weighted EER & $\begin{array}{l}\text { Control drug (standard care) } \\
\text { weighted CER }\end{array}$ & $R B I(95 \% C I)$ & Weighted ABI (CI) & $N N T(C I)$ \\
\hline Important improvement & $89 \%$ & $87 \%$ & $2 \%(-13$ to 7$)$ & $2 \%(-11$ to 7$)$ & NS \\
\hline & Zuclopenthixol EER & Control drug (haloperidol) CER & $R B I(C I)$ & $A B I$ & $N N T(C I)$ \\
\hline Sedated at 4 hours & $30 \%$ & $5 \%$ & $500 \%(8$ to 3543$)$ & $25 \%$ & $4(2$ to 59$)$ \\
\hline
\end{tabular}

†NS = not significant. Other abbreviations defined in glossary; RBI, ABI, NNT, and CI calculated from data in article.

\section{Commentary}

Major difficulties are encountered in studying the management of psychiatric emergencies, especially using randomised controlled trials (RCTs). This is reflected in the dearth of RCTs on the use of zuclopenthixol acetate in extremes of behavioural disturbance. Only 5 studies were considered suitable for inclusion in the metaanalysis by Fenton $e t$ al and, inevitably, each of these had major methodological flaws. None made fully clear the randomisation process, and 3 of the studies excluded patients from analysis after randomisation because of protocol non-compliance.

The pooled data showed no substantial improvement in psychotic symptoms at the end of the brief follow up periods, which is hardly a surprising result given that an expectation of instant antipsychotic effect does not make pharmacological sense. Sedation-probably the most desired effect-was evaluated using different instruments across the studies. One study appeared to show a statistically and clinically significant increase in sedation with zuclopenthixol acetate but this was compared with oral haloperidol, an unlikely choice in an emergency situation.

Lack of information about the effects of the different medications on numbers of aggressive incidents is disappointing because violence and threats of violence are among the most common indications for zuclopenthixol acetate. This drug cannot be given more than once in 24 hours, therefore frequency of injections-surely one of the most important outcome measures for patients-cannot be properly compared. In the absence of adequate data from RCTs, clinicians will continue to employ their knowledge of basic pharmacokinetics in choosing this medication.
This systematic review did not show superiority for zuclopenthixol acetate over other drugs in acutely disturbed psychotic patients, but no evidence of benefit is not evidence of no benefit. We agree with Fenton $e t$ al that recommendations on the use of zuclopenthixol acetate in preference to standard treatment should be viewed with caution. But it will remain a valuable option for severe behavioural disturbance that has not responded to other shorter acting medications. Clearly, more research is required, but we would question whether it is feasible to conduct an ethical RCT which can be generalised to such complex and highly charged clinical situations.

Seamus McNulty, MRCPsych Anthony J Pelosi, FRCP, MRCPsych Hairmyres Hospital Glasgow, UK 